\title{
Overview
}

\section{Current topics of S-1 at the 74th Japanese Gastric Cancer Congress}

\author{
TSUNEO SASAKI \\ Department of Chemotherapy, Tokyo Metropolitan Komagome Hospital, 3-18-22 Honkomagome, Bunkyo-ku, Tokyo 113-5677, Japan
}

\begin{abstract}
S-1 (TS-1 $\left.{ }^{\circledR}\right)$-related studies presented at the 74th Japanese Gastric Cancer Congress are reviewed. Of the papers presented at this conference, 35 were related to $S-1$. In the panel discussion on the therapeutic significance of oral fluoropyrimidines in gastric cancer, 9 papers were related to $S-1$ (sensitivities to oral fluoropyrimidines, 2 papers; clinical results of treatment with $S-1,5$ papers; and combination therapy with S-1, 2 papers). In the general presentations, there were 26 papers on S-1 related-subjects (clinical studies or clinical practice of $S-1,12$ papers; case reports, 3 papers; basic studies on animal models of peritoneal metastasis, 2 papers; and combination therapy with $S-1,9$ papers). Several studies showed that $\mathrm{S}-1$ was basically as effective against tumors in postmarketing surveillance in clinical practice as in phase II studies at the time of its development, including a report of a patient with complete response to $S-1$. Some reports suggested the possibility of using $\mathrm{S}-1$ in neoadjuvant chemotherapy and postoperative adjuvant chemotherapy. The usefulness of S-1 in combination chemotherapy was also suggested in several reports. These results indicate that $S-1$ is a key drug that can be used in first-line treatment of gastric cancer. It will be necessary to accumulate evidence based on data from clinical trials and clinical applications in the future.
\end{abstract}

Key words 74th Japanese Gastric Cancer Congress · S-1 · Overview

\section{Introduction}

Oral fluoropyrimidines, which hold the most important position in chemotherapy on an outpatient basis for gastric cancer, are widely used for advanced disease and as adjuvant chemotherapy. S-1, a new oral antitumor agent, was designed based on the theory of biochemical

Offprint requests to: T. Sasaki

Received: September 30, 2002 / Accepted: February 6, 2003 modulation of 5-fluorouracil (5-FU) [1-4]. In S-1, tegafur (FT) is combined with two classes of enzyme inhibitor, 5- chloro-2, 4-dihydroxypyridine (CDHP) and potassium oxonate (Oxo), at a molar ratio of FT:CDHP:Oxo = 1:0.4:1 [5]. S-1 achieved a response rate of $44.6 \%(45 / 101)$ in gastric cancer patients in late phase II studies [6,7]. On the strength of such excellent results in phase II studies, gastric cancer as an indication for the use of S-1 was permitted, in March 1999, and its commercial use became possible.

The 74th Japanese Gastric Cancer Congress, chaired by Dr. Yosino, was held from February 7 to 9, 2002, in Tokyo. The conference included 7 special lectures, 2 symposia, 3 video symposia, 2 panel discussions, 3 workshops, and 300 general presentations (poster sessions). Of the papers presented at this conference, 35 were related to S-1. Particularly in the "Panel discussion on the therapeutic significance of oral fluoropyrimidines in gastric cancer", all the presentations were related to S1. The following is an overview of S-1-related studies presented at this conference, with special reference to those at the panel discussion.

\section{Panel discussion: the therapeutic significance of oral fluoropyrimidines in gastric cancer}

The panel discussion for this conference was planned, and cochaired by the author and Dr. Maehara (Kyushu University). Nine papers at this symposium, all of them related to $\mathrm{S}-1$, presented updated basic and clinical evidence on this agent (Table 1).

Sensitivity to oral fluoropyrimidines, mainly $S$-1, and related factors: two papers

Usuki reported that, in a study of 80 postoperative gastric cancer patients, both UFT (uracil + tegafar) and S1 , dihydropyrimidine dehydrogenase (DPD) inhibitory 
Table 1. S-1 related reports in Panel Discussion

\begin{tabular}{|c|c|c|}
\hline No. & Title & Reporter \\
\hline PD2-01 & Dihydropyrimidine dehydrogenase activity in gastric cancer and DPD inhibitory fluoropyrimidines & Usuki \\
\hline PD2-02 & Prediction of oral fluoropyrimidine sensitivity by metabolic, catabolic and target enzymes & Fujiwara \\
\hline PD2-03 & Personalized chemotherapy in gastric carcinoma & Yoshida \\
\hline PD2-04 & Long-term control of advanced and recurrent gastric cancer by S-1 & Cho \\
\hline PD2-05 & Efficacy and safety of S-1 in patients with advanced or recurrent gastric cancer & Imamura \\
\hline PD2-06 & Neoadjuvant chemotherapy for scirrhous gastric cancer — with special reference to S-1 & Kinoshita \\
\hline PD2-07 & Clinical practice of S-1 for advanced gastric cancer & Kawai \\
\hline PD2-08 & Experimental approach for combination therapy of S-1 and docetaxel & Takahashi \\
\hline PD2-09 & Clinical significance of S-1 and S-1+CDDP for gastric cancer & Baba \\
\hline
\end{tabular}

S-1; Taiho Pharmaceutical (Tokyo, Japan); CDDP, cisplatin

Table 2. Response rates and median survival times (MSTs) of S-1 for gastric cancer in clinical practice (from Panel Discussion)

\begin{tabular}{lcccc}
\hline Reporter & $\begin{array}{c}\text { Prior } \\
\text { chemotherapy }\end{array}$ & $\begin{array}{c}\text { No. of } \\
\text { patients }\end{array}$ & $\begin{array}{c}\text { Response } \\
\text { rate }(\%) *\end{array}$ & $\begin{array}{c}\text { MST } \\
\text { (days) }\end{array}$ \\
\hline Kawai & No & 51 & 43 & 337 \\
Cho & No/Yes & 69 & 38 & 336 \\
Yoshida & No/Yes & 40 & 44 & NR \\
\hline
\end{tabular}

* Evaluable cases

NR, Not reported

fluoropyrimidines (DIF), were useful in the treatment of patients with low DPD activity and that S-1 was especially useful in patients with high DPD activity. Fujiwara, who conducted a basic study using nude mice, reported on the selection of indications for oral fluoropyrimidines in view of thymidylate synthase (TS), DPD, and thymidine phosphorylase (TP) enzyme activities.

\section{Clinical results of treatment with $S$-1 alone: five papers}

Cho, Kawai, and Yoshida reported the results of clinical practice in the treatment of gastric cancer with S-1 alone. Cho reported a survival benefit among patients who received longterm administration of S-1. Kawai reported that patients who received an initial dose of S1 showed a 2-year survival rate of $35 \%$, indicating the high efficacy of S-1. Yoshida emphasized that treatment with $\mathrm{S}-1$ alone produced better results than treatment with a low dose of cisplatin (CDDP) plus 5-FU at his facility. Yoshida also indicated the possibility of personalized chemotherapy, based on an efficacy prediction model using a statistical technique. Table 2 shows a summary of the response rates and median survival times (MSTs) of these three reports.

Imamura reported on the results of a clinical study for curing gastric cancer with S-1 alone and suggested a modified schedule to reduce adverse reactions. Kinoshita reported a pilot study of preoperative chemo- therapy with S-1 for scirrhous gastric cancer. Given these study results, the Japan Clinical Oncology Group (JCOG) is currently carrying out a phase II study of S-1 preoperative chemotherapy for scirrhous gastric cancer.

\section{S-1 therapy combined with other drugs: two papers}

Takahashi reported the results of a study on the antitumor effect and toxicity of combination chemotherapy of S-1 with docetaxel using nude rats. An increased antitumor effect in the slight toxicity range was demonstrated. Baba reported the therapeutic outcome for advanced gastric cancer patients treated with a combination of S1 and CDDP. According to the Ohtsu regimen [8], S-1 was administered orally at $80 \mathrm{mg} / \mathrm{m}^{2}$ per day twice a day for 21 consecutive days and CDDP was infused at $60 \mathrm{mg} / \mathrm{m}^{2}$ over $2 \mathrm{~h}$ on day 8 . An assessment of nine evaluable cases showed a response rate of $56 \%$. Baba stated that this combination therapy was the first choice for patients with advanced or recurrent gastric cancer and that it was suitable for preoperative chemotherapy.

Finally, Kaibara gave a special lecture. He reported an investigation of the relationship between the prognosis of patients who received oral fluoropyrimidines as postoperative adjuvant chemotherapy and the prognosis of those who also received 5-FU metabolic and catabolic enzymes. He presented results for some patients who responded to S-1 and also described the prospects for future S-1 chemotherapy.

\section{General presentations}

In the "General presentations," 26 papers on S-1 related subjects were presented in posters (Table 3). Twelve papers were about the clinical study of S-1 alone or the results of clinical practice with S-1. Three papers were case reports, including the report of a patient with a complete response to S-1. Nearly all the papers described the clinical effects of S-1 on advanced or recurrent gastric cancer, suggesting the usefulness of S-1 in 
Table 3. S-1 related reports in General Presentations

\begin{tabular}{|c|c|c|}
\hline No. & Title & Reporter \\
\hline P-042 & Experience of chemotherapy using S- 1 for advanced and recurrent gastric cancer & Nakamura \\
\hline $\mathrm{P}-063$ & A case of advanced gastric cancer improved by S-1 & Nagai \\
\hline P-064 & Clinical efficacy of S-1 for treatment of advanced gastric cancer & Nakamura \\
\hline P-065 & A case of advanced gastric cancer treated with S-1 and CPT-11 & Yamashita \\
\hline P-075 & Preoperative chemotherapy with S-1 and low dose CDDP for advanced gastric cancer & Saikawa \\
\hline P-078 & Effect of administration of CPT-11 combined with CDDP on S-1 tolerated gastric cancer & Sugiura \\
\hline P-079 & FLEP/S-1 therapy for advanced gastric cancer & Mochizuki \\
\hline P-080 & Effectiveness of S-1 for gastric cancer: emphasis on effective cases & Sato \\
\hline P-081 & $\begin{array}{l}\text { Clinical effect and adverse reactions of S-1 administration before surgery for advanced gastric } \\
\text { cancer }\end{array}$ & Chochi \\
\hline P-082 & S-1/CDDP therapy for advanced gastric cancer & Nakamura \\
\hline P-083 & Chemotherapy with S-1 and CDDP for advanced gastric cancer & Michiura \\
\hline P-084 & The effect of S-1 and combined chemotherapy of CDDP/S-1 for highly advanced gastric cancer & Konno \\
\hline P-090 & New therapeutic experience of $S-1$ for reduction of adverse reactions & Kimura \\
\hline $\mathrm{P}-091$ & A case of gastric cancer with liver metastasis and LN metastasis treated with S-1/CDDP & Maruyama \\
\hline P-167 & Evaluation of the efficacy of S-1 on peritoneal metastasis of gastric cancer using an animal model & Mori \\
\hline P-168 & Inhibition of peritoneal micrometastasis of GFP-tagged gastric cancer cells by S-1 & Mochizuki \\
\hline $\mathrm{P}-175$ & Effects of S-1 on recurrent gastric cancer and consideration of the indications for administration & Yamada \\
\hline P-185 & Umbilical metastasis of gastric cacer & Nakasato \\
\hline P-187 & $\begin{array}{l}\text { A case in which S-1+CDDP therapy worked effectively on DIC due to multiple bone } \\
\text { metastasis of gastric cancer }\end{array}$ & Yoshioka \\
\hline P-238 & Home therapy using S-1 in combination with enteral nutrition support for gastric cancer patients & Sano \\
\hline P-279 & $\begin{array}{l}\text { Successful treatment for gastric cancer associated with multiple liver metastases with S-1 } \\
\text { followed by curative gastrectomy }\end{array}$ & Suzuki \\
\hline P-280 & Three cases with effective response to $\mathrm{S}-1$ for inoperable gastric cancer & Urano \\
\hline P-281 & Improvement in the survival of patients with advanced gastric cancer by S-1 & Watanabe \\
\hline P-282 & Significance of S-1 for advanced or recurrent gastric cancer & Ohashi \\
\hline $\mathrm{P}-283$ & A clinical study of S-1 in patients with advanced and recurrent gastric cancer & Teruya \\
\hline P-284 & Feasibility and efficacy of S-1 adjuvant chemotherapy for resectable stage IV gastric cancer & Fujitani \\
\hline
\end{tabular}

CPT-II, Irinotecan; FLEP, 5-FU + leucovorin + etoposide + CDDP; LN, lymph node; GFP, green fluorescent protein; DIC, disseminated intravascular coagulation

patients with advanced or recurrent gastric cancer. Fujitani et al. reported on the safety and efficacy of S-1 for stage IV cancer, and showed the possibility that S-1 contributes to the survival of patients when used in adjuvant chemotherapy for stage IV gastric cancer.

There were two reports of basic studies, both about S-1 in animal models of peritoneal metastasis. Mori demonstrated the usefulness of S- 1 for peritoneal dissemination, using an experimental model of peritoneal dissemination of gastric cancer. Mochizuki et al. presented an experimental study in a model of peritoneal dissemination of green fluorescent protein (GFP)tagged human gastric cancer. They reported that the inhibitory effect of S-1 on peritoneal disseminated metastasis improved as the number of viable cancer cells in the peritoneal cavity decreased, and that S-1 improved survival prognosis if the number of cancer cells was small.

Papers on combination chemotherapy with S-1 included six reports on the results of clinical studies or the results of clinical practice at individual medical centers, and three case reports. Combination chemotherapy involved S-1 + CDDP in seven reports (the majority), S$1+$ irinotecan (CPT-11) in one report, and 5-FU + leucovorin + etoposide + CDDP $($ FLEP $)+\mathrm{S}-1$ in one report.

\section{Summary and future prospects}

The S-1-related studies presented at the 74th Japanese Gastric Cancer Congress have been summarized. Several studies showed that S-1 was generally as effective against tumors in the postmarketing surveillance in clinical practice as in phase II studies at the time of its development. Because S-1 is an oral preparation, it can be used on an outpatient basis and should be beneficial from the viewpoint of the patient's quality of life (QOL). These results indicate that S-1 is a key drug that can be used in the first-line treatment of gastric cancer. Some reports suggested the possibility of using S- 1 in neoadjuvant chemotherapy and postoperative adjuvant chemotherapy. Thus, it is necessary to verify the usefulness of S-1 in a large-scale prospective randomized trial. The usefulness of S-1 in combination chemotherapy was also suggested by several reports, although only preliminary results have been obtained so far. Further clinical studies must be conducted to establish combination 
chemotherapy involving S-1, CDDP, CPT-11, paclitaxel, and docetaxel.

Because S-1 is expected to become the standard drug for the treatment of gastric cancer, it will be necessary to build up evidence by further accumulation of data from clinical trials and clinical practice in the future.

\section{References}

1. Shirasaka T, Nakano K, Takechi T, Satake H, Uchida J, Fujioka A, et al. Antitumor activity of $1 \mathrm{M}$ tegafur- $0.4 \mathrm{M}$ 5-chloro-2,4dihydropyrimidine-1 M potassium oxonate (S-1) against human colon carcinoma orthotopically implanted in nude rat. Cancer Res 1996;56:2602-6.

2. Shirasaka T, Simamoto Y, Fukushima Y. Inhibition by oxonic acid of gastrointestinal toxicity of 5-fluorouracil without loss of its antitumor activity in rats. Cancer Res 1993;53:4004-9.

3. Fukushima M, Satake H, Uchida J, Shimamoto Y, Kato T, Takechi T, et al. Preclinical antitumor efficacy of S-1: a new oral formulation of 5-fluorouracil on human tumor xenografts. Int J Oncol 1998;13:693-8.
4. Kato T, Shimamoto Y, Uchida J, Ohshimo H, Abe M, Shirasaka T, et al. Possible regulation of 5-fluorouracil-induced neuro- and oral toxicities by two biochemical modulations consisting of S-1, a new oral formulation of 5-fluorouracil. Anticancer Res 2001;21:170512.

5. Shirasaka T, Shimamoto Y, Ohshima H, Yamaguchi M, Kato T, Yonemura K, Fukushima M. Development of a novel form of an oral 5-fluorouracil derivative (S-1) directed to the potentiation of the tumor selective cytotoxicity of 5-fluorouracil by two biochemical modulators. Anticancer Drugs 1996;7:54857.

6. Sakata Y, Ohtsu A, Horikoshi N, Sugimachi Y, Mitachi Y, Taguchi $\mathrm{T}$. Late phase II study of novel oral fluoropyrimidine anticancer drugs S-1 (1 M tegafur- $0.4 \mathrm{M}$ gimestat- $1 \mathrm{M}$ otastat potassium) in advanced gastric cancer patients. Eur J Cancer 1998;34:171520.

7. Koizumi W, Kurihara M, Nakano S, Hasegawa K. Phase II study of $\mathrm{S}-1$, a novel oral derivative of 5-fluorouracil, in advanced gastric cancer. Oncology 2000;58:191-7.

8. Ohtsu A, Boku N, Nagashima F, Koizumi W, Tanabe S, Saigenji K, et al. A phase I/II study of S-1 plus cisplatin (CDDP) in patients (pts) with advanced gastric cancer (ACG). Proc Am Soc Clin Oncol 2001;20:165a. 